\title{
REPRESENTING UNCERTAINTY IN PROPERTY VALUATION THROUGH A BAYESIAN DEEP LEARNING APPROACH
}

\author{
Changro Lee \\ Department of Real Estate \\ Kangwon National University \\ e-mail: spatialstat@kangwon.ac.kr \\ Keith Key-Ho Park \\ Department of Geography \\ Seoul National University \\ Institute for Korean Regional Studies \\ e-mail:khp@snu.ac.kr
}

\begin{abstract}
Although deep learning-based valuation models are spreading throughout the real estate industry following the artificial intelligence boom, property owners and investors continue to doubt the accuracy of the results. In this study, we specify a neural network for predicting house prices. We suggest a standard feed-forward network with two hidden layers, and show that it is sufficiently reasonable to apply its prediction to real-world projects such as property valuation. In addition, we propose a Bayesian neural network for describing uncertainty in house price predictions while providing a means to quantify uncertainty for each prediction. We choose Gangnam-gu, Seoul for the analysis, and predict house prices in the area using both networks. Although the Bayesian neural network did not perform better than the conventional network, it could provide a tool to measure the uncertainty inherent in predicted prices. The findings of this study show that a Bayesian approach can model uncertainty in property valuation, thereby promoting the adoption of deep learning tools in the real estate industry.
\end{abstract}

Key words: deep learning, Bayesian neural network, uncertainty, property valuation.

JEL Classification: E37, L85, R00, C11, C45.

Citation: Lee, C., \& Park, K. K.-H. (2020). Representing uncertainty in property valuation through a bayesian deep learning approach. Real Estate Management and Valuation, 28(4), 15-23.

DOI: https://doi.org/10.1515/remav-2020-0028

\section{Introduction}

Although deep learning tools, such as neural networks, are used extensively in a variety of fields, such as medicine, healthcare and automobile business, conventional deep learning tools for regression and classification have not paid due attention to model uncertainty (Gal \& Ghahramani, 2016). Representing model uncertainty, however, is indispensable for applications in the real-world (Ghahramani, 2015). In real-world tasks, what is desired is not simply results, but also assurance of the results. For instance, when spotting invasive cancer cells in the brain, one would want not to only detect cancerous cells but also express confidence that the lesion is cancerous and not benign. A crucial part of intelligence is reserving one's judgment when one is uncertain. Thus, it is surprising that measuring uncertainty is not in the aim in the case of many deep learning applications. 
The conventional neural network would present a predicted value even if it were given input that is completely unrelated to what it has been trained with. If the new input data is something the network has never seen, the uncertainty will be high, and the network should produce an answer tagged with a message such as "I do not know what this is about."

In this study, we develop the model necessary to represent uncertainty in deep learning and apply it to property valuation. Property valuation is a key part of real estate in fields such as brokerages, tax assessment, collateral loans, and property portfolio management. We develop a hedonic pricing model to predict house prices from house transaction data. We implement a standard feed-forward neural network, and then propose a Bayesian neural network as an alternative to quantifying uncertainty over the predicted prices. The conventional deep neural network which does not maintain Bayesian posterior updates will be referred to as Artificial Neural Network (ANN, for short), and Bayesian neural network as BNN, respectively.

We choose Gangnam-gu, Seoul as a study area and predict house prices in the area using both of the networks. We then compare the outputs obtained from ANN and BNN. Lastly, we discuss the advantage of accounting for uncertainty when estimating property prices in the setting of property valuation.

This study is the first attempt to describe uncertainty in property valuation through a Bayesian deep neural network. Deep learning-based valuation models are gaining in popularity nowadays, but they are not sufficient to convince property owners and investors to trust the results (Conway, 2018). This study suggests a Bayesian approach to model uncertainty in property valuation, promoting the adoption of deep learning tools in real estate.

This study proceeds from a review of uncertainty in property valuation and Bayesian probability theory related to uncertainty; the third section explains the data used and network architectures. Then, the main results and implications are provided in the fourth section, and finally, a summary of the study and conclusions are presented.

\section{Literature review}

\subsection{Uncertainty in property valuation}

Despite the widespread success of deep learning, ANNs lack probabilistic considerations. This is an issue in applications where representing uncertainty is of critical importance (Ghahramani, 2015). Medical diagnosis would be a good example of a situation where confidence is a key factor in decision-making (Johnson et al., 2016). Property valuation is also one of the application fields where describing uncertainty plays a critical role when making a decision, such as approving real estate loans. Kucharska-Stasiak (2013) stated that uncertainty in property valuation is exceptionally high due to both the characteristics of real estate (e.g., fixed location, long useful life, variations in physical features) and the characteristics of the real estate market (e.g., low efficiency, low elasticity of supply and demand). According to his argument, the more individualized the physical characteristics of a property and the lower the market trade volumes are, the more difficult it is to accurately estimate property value.

There will always be a degree of uncertainty in any valuation, and this rises sharply when a combination of the rare characteristics of real estate and the thin market lead to the model being unable to predict with confidence of accuracy (French \& Gabrielli, 2004). A unique house with extensive balconies and a spacious grassy area in a neighborhood where plank houses with coarse, thatch roofs are common would be a good example of a property with rare characteristics, and it is certainly difficult to estimate the property's value accurately. As expected, a low level of market activity will also lead to a high degree of uncertainty in price estimation due to a lack of evidence for market value. Thus, property valuation should reflect this uncertainty when the subject property is a real estate of rare type and the neighborhood has shown little trade activity in recent years.

In valuations prepared for insurance or loans, predicted prices are usually provided as single figures. However, from both the point of view of valuation theory and practice, a single figure is a myth (Kucharska-Stasiak, 2013). Although the valuer needs to provide a single figure, a description should be developed to explain the uncertainty of the final figure (Mallinson \& French, 2000).

If the process of identification and description of uncertainty was provided, it would greatly assist many decision-makers and improve the credibility of the valuation work (Mallinson \& French, 2000). In fact, a few attempts to explain the uncertainty in valuation have been made, such as employing the 
theory of games (Meszek, 2007) and using a scenario analysis (French, 2001). However, the mathematical quantification of uncertainty within a valuation is rarely conducted or conveyed to the user in any coherent form (Joslin, 2005).

\subsection{Bayesian neural network}

ANN can be viewed as a generalization of linear models that perform multiple stages of processing to come to a decision. But it lacks probabilistic considerations. To complement this, ANN is reformulated as BNN to capture uncertainty in the target value. In this framework, network weights belong to prior distributions with parameters $\theta$. Posterior distributions are conditioned on the data following the Bayes theorem:

$$
p(\theta \mid D)=\frac{p(D \mid \theta) p(\theta)}{p(D)}
$$

where: $\mathrm{D}$ is the data.

The key idea in the Bayesian perspective is that everything has a probability distribution attached to it, including model parameters (weights and biases in neural networks). In an ANN approach, there are fixed weights and biases that determine how an input is transformed into an output. In a BNN perspective, all weights and biases have a probability distribution attached to them. These probability distributions describe the uncertainty in weights and can be used to estimate uncertainty in predictions.

While useful in terms of the theoretical perspective, Equation (1) is infeasible to compute. The evidence term in the denominator amounts to the integral over all possible values of the network weights:

$$
p(D)=p(x, y)=\int p(x, \theta) p(\theta) d \theta
$$

where the data $\mathrm{D}$ can be written as $x, y$ input-output pairs for supervised learning.

Calculating the evidence term is difficult due to computational expensiveness. Instead, various alternative approaches have been presented to approximate these posterior distributions. Samplingbased approaches are widely used under the name of Monte-Carlo methods (Neal, 2012).MetropolisHastings in particular is a popular algorithm for Monte-Carlo sampling.

However, for modern Bayesian deep learning architectures, such as a neural network with millions of parameters, Monte-Carlo sampling is still slow to converge and may not discover the full posterior distribution. Research in the area has concentrated on finding more efficient inference methods including stochastic gradient Langevin dynamics (Welling \& Teh, 2011), expectation propagation (Hasenclever et al. 2017), and variational Bayes (Graves, 2011; Wang \& Blei, 2019).

In this paper, we adopt variational Bayes as an efficient way to describe uncertainty over property price predictions. The core of variational Bayes methods is that, since we cannot exactly compute the posterior, we can find the closest probability distribution to it that is "easy to approach." By "easy to approach" we mean a distribution (for example, a normal distribution) that can be represented by a few sets of parameters, like mean or variance. Thus, after random initialization of those parameters in an "easy to approach" distribution, gradient descent is implemented and parameters of the distribution (like mean or variance) are updated each time to verify if the resultant distribution is closer to the target posterior. We do not, however, know whether the resulting distribution is closer to the target posterior since the posterior is precisely what we want to calculate. But we do not need the exact posterior to find closeness between it and the other "easy to approach" distribution. We actually optimize closeness between the two distributions through evidence lower bound or ELBO (Jang \& Lee, 2019). It is a loss function, similar to mean squared error (MSE) in a non-Bayesian neural network.

\section{Data and network architecture}

\subsection{Gangnam-gu, Seoul}

This study estimates the single-family house prices in Gangnam-gu, Seoul, S. Korea. Seoul is the capital of S. Korea, with a population of about 10 million distributed over $605.2 \mathrm{~km}^{2}$ of administrative areas. The population density is 16,492 persons per $\mathrm{km}^{2}$ (Seoul, 2017), one of the highest amongst top global cities. Gangnam-gu, one of the 25 local government districts in Seoul, is well known for its high standard of living and heavily concentrated wealth. Most importantly, Gangnam-gu is widely known for its extremely high property prices. 


\section{S sciendo}

\subsection{Dataset}

The dataset consists of 488 houses sold between 2011 and 2014. It is divided into 366 training samples $(75 \%)$ and 122 test samples $(25 \%)$ in a random manner. Table 1 shows the descriptive statistics for the dataset used in the study, and Figure 1 shows the locations of the 488 single-family houses.

Table 1

Descriptive statistics $(\mathrm{n}=488)$

\begin{tabular}{ccccc}
\hline & Min. & Mean & Median & Max. \\
\hline sales prices $($ million KRW) & 45 & 2,221 & 1,988 & 13,000 \\
\hline site area $\left(\mathrm{m}^{2}\right)$ & $7.3^{*}$ & 239.7 & 224.6 & 908.3 \\
\hline building area $\left(\mathrm{m}^{2}\right)$ & 65.8 & 358.6 & 316.8 & $2,088.0$ \\
\hline age (year) & 1 & 24.4 & 24 & 42 \\
\hline
\end{tabular}

* a site where the ownership is shared by two or more owners, indicating that one of the shares was sold.

Source: own study.

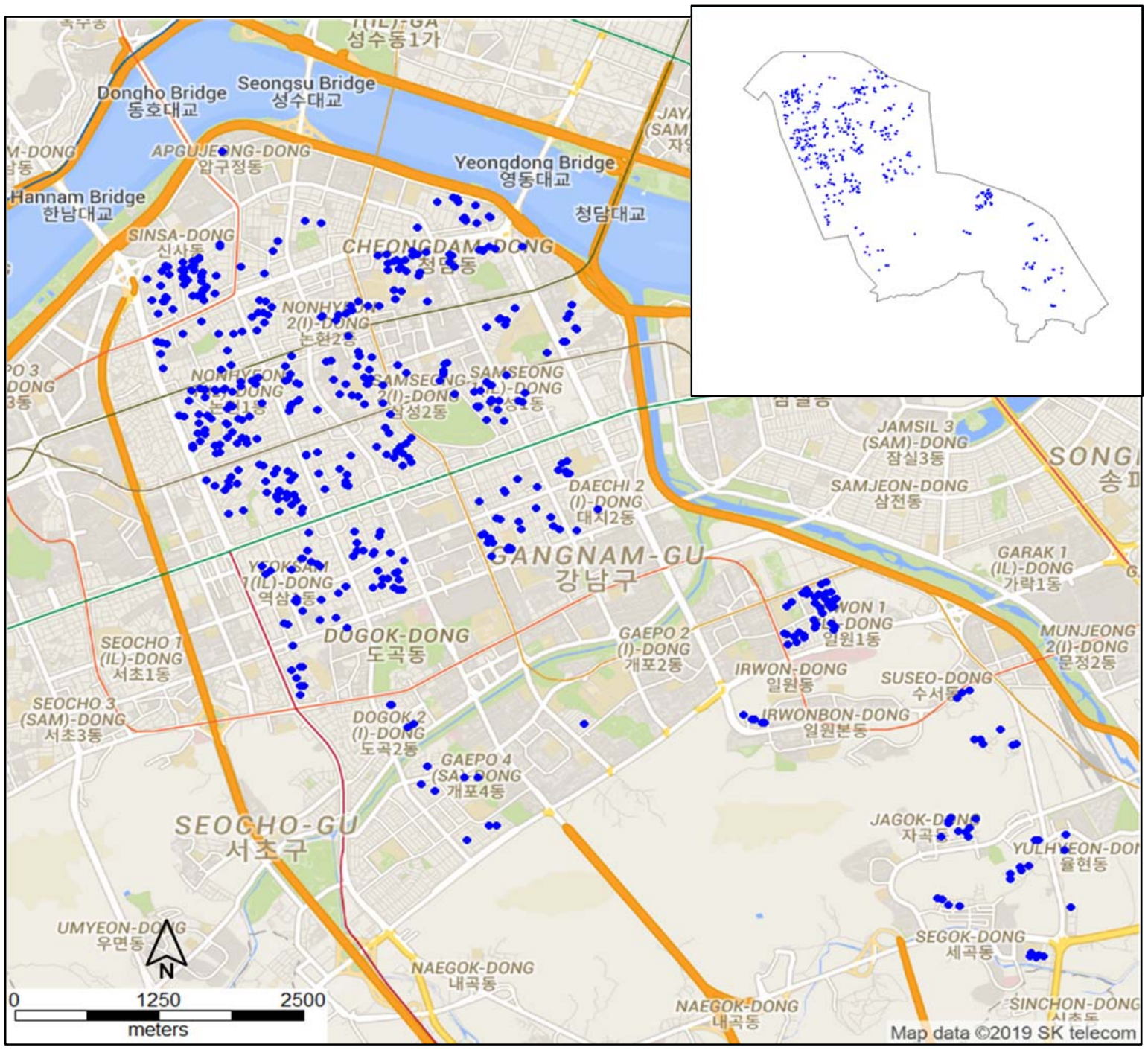

Fig. 1. Sample locations in Gangnam-gu, Seoul. Source: Background map from Google Maps and SK telecom map data. 
Explanatory variables are chosen as the following: road width $(\mathrm{m})$, building area $\left(\mathrm{m}^{2}\right)$, site area $\left(\mathrm{m}^{2}\right)$, year traded, property age (year), zone, slope, site shape, site bearing, building structure, neighborhood characteristics, and each property's longitude and latitude. Factors affecting house prices could be enumerated infinitely; therefore, the choice of explanatory variables is always compromised between real estate market theory and data collectability. We chose the above listed variables because they were available during the data collection process. Moreover, they play an important role in shaping house prices. For example, road width is one of the main factors that most valuers take into account when estimating property prices; longitude and latitude, that is, the geographical location of a house, is another factor that property valuers consider when conducting valuation. The total number of explanatory variables is 13, and some were collected as categorical variables. We convert the categorical variables into ordinal variables, a sort of pseudo-continuous variable, so that they can be fed into the neural network efficiently. Domain knowledge from valuation experts is borrowed for this conversion ${ }^{1}$. Table 2 shows conversions of categorical variables used in the network. All the explanatory variables are standardized to have zero mean and one standard deviation. Sales prices (unit: million KRW) are normalized by dividing the raw figure by the maximum value of the sales prices.

Table 2

Conversion of categorical variables to ordinal ones

\begin{tabular}{cc}
\hline Variable & Conversion \\
\hline Zone & Environment preservation $\rightarrow 1, \ldots$, Commerce $\rightarrow$ S \\
\hline Slope & Sloping $\rightarrow 1$, Level $\rightarrow 2$ \\
\hline Site shape & Irregular $\rightarrow 1$, Rectangular $\rightarrow 2$ \\
\hline Site bearing & North $\rightarrow 1, \ldots$, South $\rightarrow 3$ \\
\hline Building structure & Steel pipe $\rightarrow 1, \ldots$, Reinforced concrete frame $\rightarrow 3$ \\
\hline Neighborhood characteristics & Farmland and forest $\rightarrow 1, \ldots$, Downtown $\rightarrow 6$ \\
\hline
\end{tabular}

\subsection{Network architectures}

A fully-connected architecture with two hidden layers is adopted. The number of units in each hidden layer is set to be 10, which is between the number of input variables (13) and output variables (1). This architecture produced the best results in most trials and was applied to both ANN and BNN.

Both networks were trained with Keras, one of the widely used deep learning libraries, and BNN is implemented following the approach described in Blundell et al. (2015).

\section{Results and discussion}

\subsection{Results}

Table 3 shows the results of training both networks. The MSE is calculated from the separately reserved test data (122 test samples), and the figure from ANN is calculated as 0.008. As for the figure from BNN, it is calculated as 0.016, which is a median value of 100 predictive simulations of each of the test data. Figure 2 shows the goodness of fit of the two networks, and the predicted values from BNN are median values from 100 predictive simulations of each of the test data. Both the MSE figures and the fit curves indicate that ANN performed better than BNN.

Table 3

Comparison of average accuracy

\begin{tabular}{|c|c|c|}
\hline & ANN & BNN \\
\hline MSE & 0.008 & 0.016 \\
\hline
\end{tabular}

Source: own study.

${ }^{1}$ Valuation manuals issued by Korea Association of Property Appraisers (KAPA) were taken into account, after which two field appraisers were consulted for assessing the relevance of this conversion. 

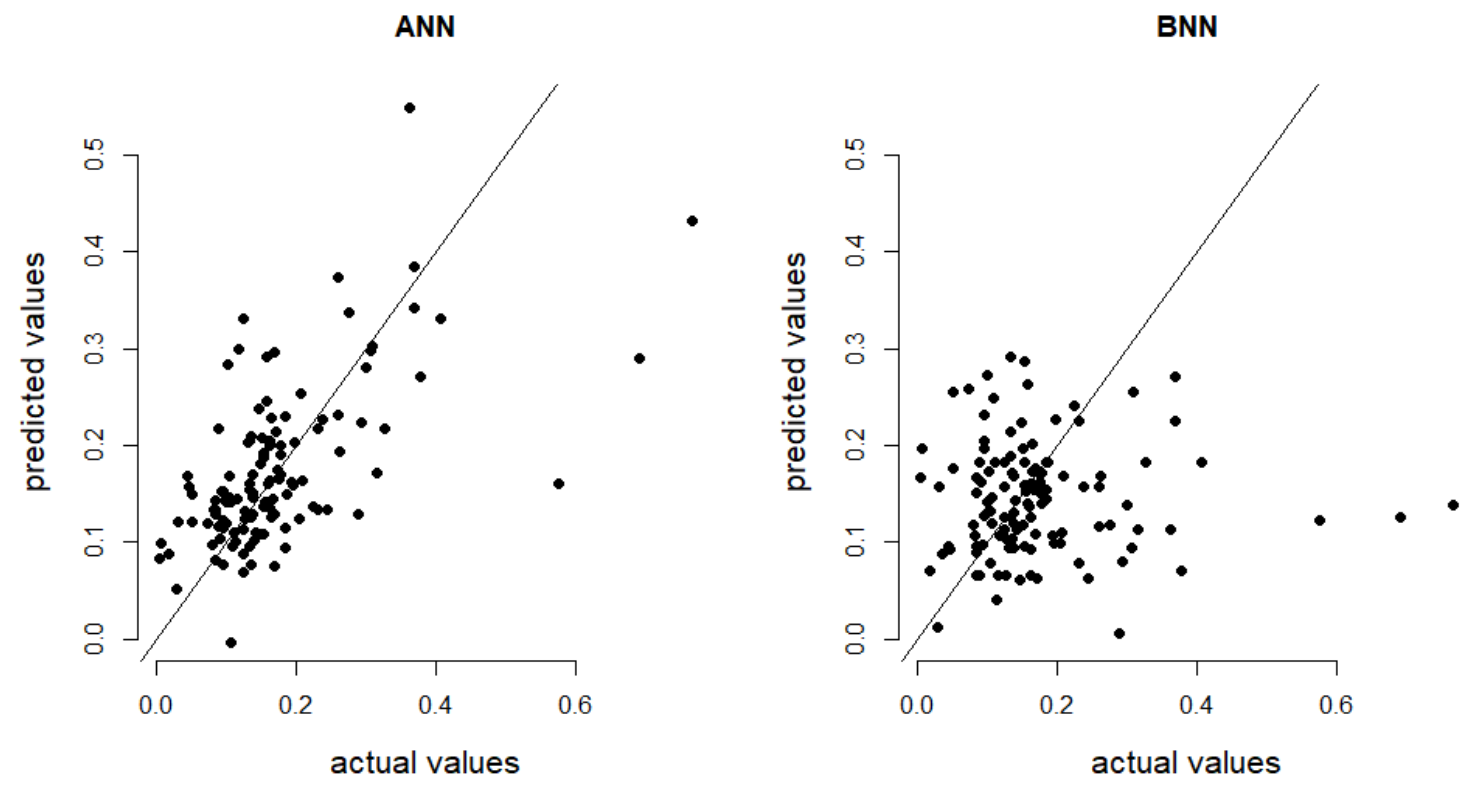

Fig. 2. Goodness of fit for models (122 samples of test data). Source: own study.

The reasons behind the BNN's poor performance seem to be related to the algorithms employed, unstable convergence and sub-optimal priors used. The computational burden for Baysian estimation is well known, and various calculation algorithms, including Metropolis-Hastings, Gibbs sampling, integrated nested Laplace approximations (INLA), among others, have been proposed. There is, however, no coherent conclusion regarding the efficiency of these algorithms. Although this study follows the algorithm used in Blundell et al. (2015), it is possible that using other algorithms would improve BNN's current performance. Unstable convergence, such as the local minima, could be pointed out as yet another reason for the current performance of BNN. Finally, parameters (weights) in BNN are influenced by the prior distributions imposed, which are not optimal in most cases. These elements seem to contribute to the current performance of BNN. However, it is difficult to generalize the result of this study, especially considering the sample size used (488 houses). Contrary results could, however, be displayed if different application areas were investigated.

In this study, the performance of ANN is considered satisfactory; hence, the conclusion that its application to real-world tasks, such as collateral valuation in financial institutes, is not problematic. Since performance of ANN is outstanding, its residuals could be used as a proxy for measuring the difficulty of predictions. That is, a large residual from ANN indicates a large prediction error, and thus, a high level of uncertainty in the predicted prices. Although BNN performed poorly compared to ANN, it has a big advantage in representing model uncertainty. We show this advantage by using the residuals from ANN.

\subsection{Analysis of uncertainty}

Uncertainty in predictions that arise from the uncertainty in weights is called epistemic uncertainty (Kendall \& Gal, 2017). This kind of uncertainty can be reduced if we obtain enough data. Thus, epistemic uncertainty would be higher in regions of little training data, and lower in regions of abundant training data in property valuation tasks. Uncertainty coming from the inherent noise in training data is an example of aleatoric uncertainty. It cannot be reduced even if more training data is given. Aleatoric uncertainty is covered by the probability distribution used to define the likelihood function, and we do not discuss this uncertainty in the present study.

Figure 3 shows uncertainty of predicted prices from the 122 test samples. The residual on the vertical axis is one from ANN. The range (left) and standard deviation (right) on the horizontal axis are calculated from 100 predictive simulations of each of the test samples through BNN. For example, if the predicted prices of the first sample of the test data vary from 0.2 to 0.5 during 100 simulations, its range value is 0.3 . This figure of 0.3 is plotted on the horizontal axis, and the corresponding residual from ANN is plotted on the vertical axis. Though we do not know the true uncertainty of 
predictions, the residuals from ANN could act as a proxy for it. As is clearly shown in Figure 3, when the range or standard deviation of predicted prices increases, the corresponding residual also increases. It can be interpreted that the $\mathrm{BNN}$ approach can effectively capture uncertainty in predictions through measures such as range or standard deviation of estimated prices from predictive simulations.
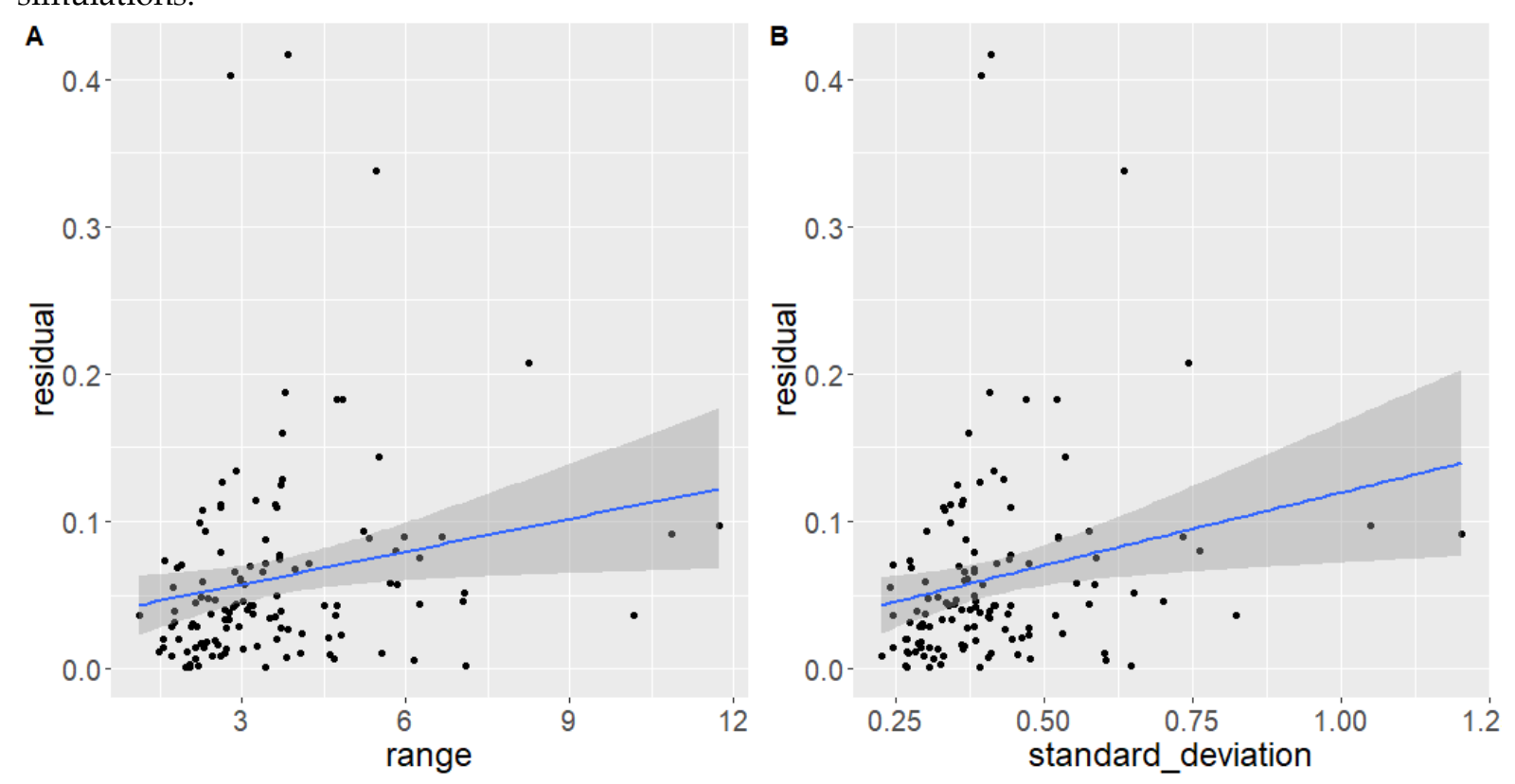

* line: linear regression fitting line, shaded area: 95\% confidence region of the regression line

Fig. 3. Prediction uncertainty in terms of range and standard deviation. Source: own study.

Figure 4 shows the locations of the test samples with their ranges of estimated prices proportional to the size of the circle. Particularly, data points with more than a range of six are displayed in the filled circles. Predicted prices are normalized ones, and thus we do not try to interpret these prices in terms of real sales price levels in Seoul. From Figure 4, it is obvious that epistemic uncertainty is higher in geographical areas of no or little training data. All the filled circles are located in the southern region of Gangnam-gu, which shows sparse distribution of sales data. It is not surprising that this area shows few sales transactions, since most of the area is hilly or mountainous. The majority of the houses displayed in filled circles are also large, luxurious homes which are difficult to find in downtown Seoul. Both, the sparce trade activity and the rare characteristics of the houses can be interpreted as leading to the high uncertainty of estimated prices.

Incorporating Bayesian uncertainties could drastically improve the applicability of deep learning approaches in tasks where confidence is crucial. With model uncertainty in our toolset, we can treat properties in thin markets or with rare characteristics explicitly. For example, approval of real estate loans may require high levels of certainty to guarantee debt collection, and predictions with high uncertainty may be sent to a human expert for on-site inspection.

\section{Conclusions}

In this study, we developed a neural network for predicting house prices. We suggested an ANN model with two hidden layers, and showed that its prediction is sufficiently accurate to be applied to real-world tasks such as collateral valuation and property tax assessment. We further proposed a BNN approach for representing uncertainty over house price predictions, providing a means to capture confidence levels for each prediction. Though BNN did not perform better than ANN, it could provide a tool to measure uncertainty in predicted prices.

We developed and trained BNN, placing red flags alongside predictions with high uncertainty. If, for example, ANN and BNN were employed together in real-world applications and the predicted price from ANN with range or standard deviation from BNN below a certain threshold were provided to a loan client, valuation accuracy and the soundness of collateral asset would be improved considerably. For instance, the acceptance criteria may be set to a 6.0 range or 0.75 standard deviation 


\section{S sciendo}

of predicted values; then, as shown in Figure 3, only the prices which have a range of less than 6.0 or standard deviation less than 0.75 would be provided to the user, reducing prediction error.

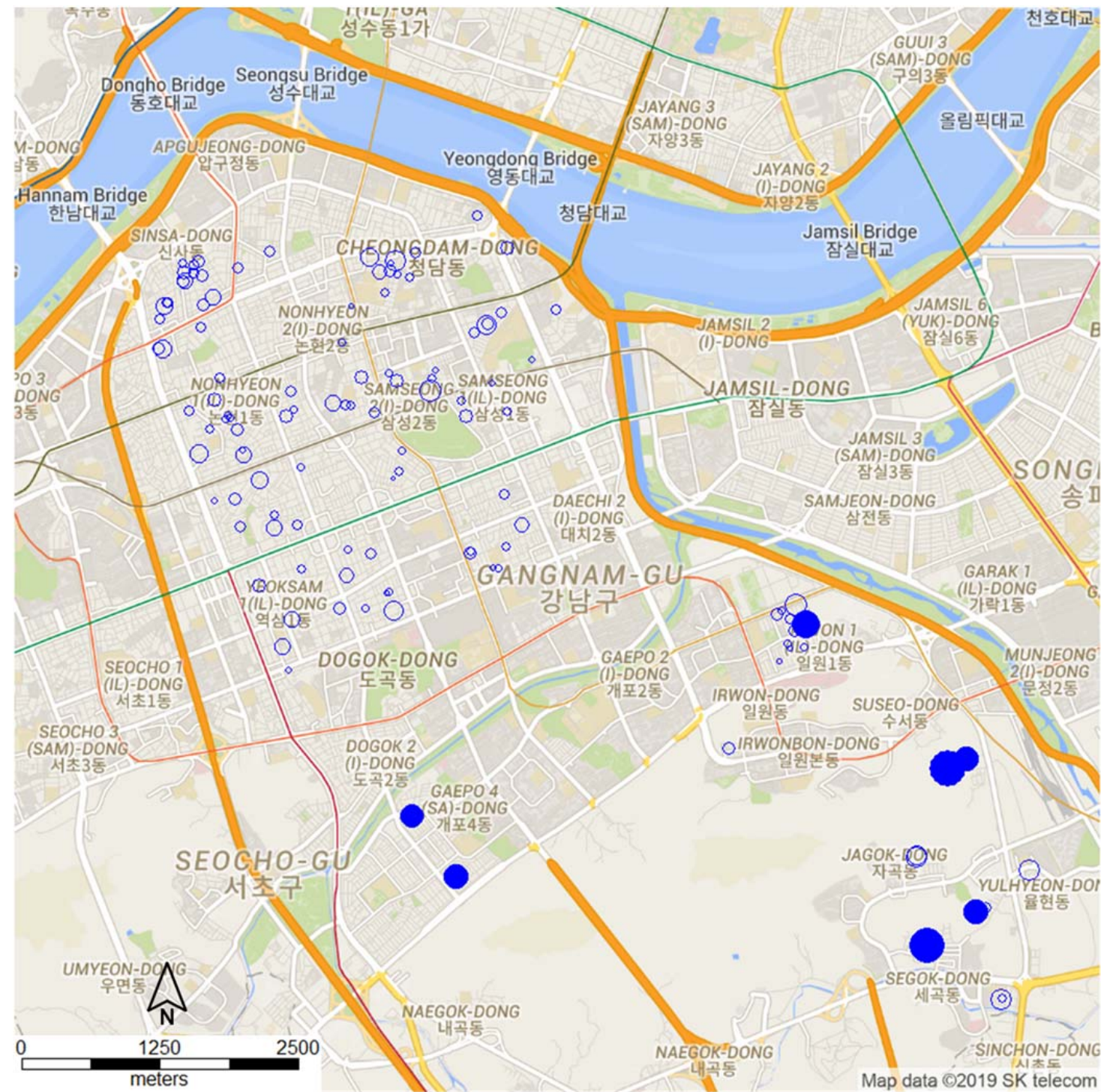

* Data points with range $>6.0$ are displayed in filled circles.

Fig. 4. Locations of test samples and prediction uncertainty attached to them. Source: Background map from Google Maps and SK telecom map data.

In short, providing only filtered or accepted predictions may become possible under the Bayesian framework, and, for this reason, the BNN model should be used in addition to the deterministic ANN model (Harper \& Southern, 2019). The approach proposed in this study could be utilized in any area where describing uncertainty is invariably necessary, and this includes property valuation, prediction of credit default risk, medical diagnosis, and collision risk assessment in autonomous vehicles. Safer use of deep learning tools is possible under the Bayesian framework, reducing the failure risk of the predictions at hand.

This study is the first attempt to train the BNN model for a property valuation problem and provide relevant uncertainty criteria. We hope this study will promote more interest in managing uncertainty of deep learning projects, including property valuation.

\section{References}

Blundell, C., Cornebise, J., Kavukcuoglu, K., \& Wierstra, D. (2015). Weight uncertainty in neural networks. arXiv preprint arXiv:1505.05424. 
Conway, J. (2018). Artificial intelligence and machine learning: current applications in real estate. Master's Thesis, Massachusetts Institute of Technology, Cambridge, Massachusetts

French, N. (2001). Uncertainty in property valuation: The pricing of flexible leases. Journal of Corporate Real Estate, 3(1), 17-27. https://doi.org/10.1108/14630010110811454

French, N., \& Gabrielli, L. (2004). The uncertainty of valuation. Journal of Property Investment \& Finance, 22(6), 484-500. https://doi.org/10.1108/14635780410569470

Gal, Y., \& Ghahramani, Z. (2016). Dropout as a bayesian approximation: Representing model uncertainty in deep learning. In international conference on machine learning (pp. 1050-1059).

Ghahramani, Z. (2015). Probabilistic machine learning and artificial intelligence. Nature, 521(7553), 452-459. https://doi.org/10.1038/nature14541 PMID:26017444

Graves, A. (2011). Practical variational inference for neural networks. In Advances in neural information processing systems, 2348-2356.

Hasenclever, L., Webb, S., Lienart, T., Vollmer, S., Lakshminarayanan, B., Blundell, C., \& Teh, Y. W. (2017). Distributed Bayesian learning with stochastic natural gradient expectation propagation and the posterior server. Journal of Machine Learning Research, 18(1), 3744-3780.

Harper, R., \& Southern, J. (2019). A Bayesian Deep Learning Framework for End-To-End Prediction of Emotion from Heartbeat. arXiv preprint arXiv:1902.03043.

Jang, H., \& Lee, J. (2019). Generative Bayesian neural network model for risk-neutral pricing of American index options. Quantitative Finance, 19(4), 587-603. https://doi.org/10.1080/14697688.2018.1490807

Johnson, A. E., Ghassemi, M. M., Nemati, S., Niehaus, K. E., Clifton, D. A., \& Clifford, G. D. (2016). Machine learning and decision support in critical care. Proceedings of the IEEE. Institute of Electrical and Electronics Engineers, 104(2), 444. https:// doi.org/10.1109/JPROC.2015.2501978

Joslin, A. (2005). An investigation into the expression of uncertainty in property valuations. Journal of Property Investment \& Finance, 23(3), 269-285. https:// doi.org/10.1108/14635780510599476

Kendall, A., \& Gal, Y. (2017). What uncertainties do we need in bayesian deep learning for computer vision? In Advances in neural information processing systems, 5574-5584.

Kucharska-Stasiak, E. (2013). Uncertainty of property valuation as a subject of academic research. Real Estate Management and Valuation, 21(4), 17-25. https://doi.org/10.2478/remav-2013-0033

Mallinson, M., \& French, N. (2000). Uncertainty in property valuation-The nature and relevance of uncertainty and how it might be measured and reported. Journal of Property Investment \& Finance, 18(1), 13-32. https:// doi.org/10.1108/14635780010316636

Meszek, W. (2007). Uncertainty phenomenon in property valuation. International Journal of Management and Decision Making, 8(5/6), 575-585. https://doi.org/10.1504/IJMDM.2007.013419

Neal, R. M. (2012). Bayesian learning for neural networks (Vol. 118). Springer Science \& Business Media.

Seoul. (2017). Seoul Statistics. Department of housing policy.

Wang, Y., \& Blei, D. M. (2019). Frequentist consistency of variational Bayes. Journal of the American Statistical Association, 114(527), 1147-1161. https:// doi.org/10.1080/01621459.2018.1473776

Welling, M., \& Teh, Y. W. (2011). Bayesian learning via stochastic gradient Langevin dynamics. In Proceedings of the 28th international conference on machine learning (ICML-11) (pp. 681-688). 